\title{
Mesotheliomas with crystalloid structures: report of nine cases, including one with oncocytic features
}

\author{
Nelson G Ordóñez \\ Department of Pathology, The University of Texas MD Anderson Cancer Center, Houston, TX, USA
}

\begin{abstract}
Although the presence of crystalloids has historically been of largely academic interest or simply an intriguing curiosity, these structures have occasionally been useful in the differential diagnosis of some tumors. Crystalloids have only rarely been reported in mesotheliomas, and their presence in these tumors has not been sufficiently investigated, nor has their potential value as an ultrastructural marker for mesothelioma been established. The finding of a case of mesothelioma in which the vast majority of the neoplastic cells contained intracytoplasmic crystalloids prompted a search for these structures in 69 consecutive cases of mesothelioma (59 epithelioid, 7 sarcomatoid, 3 mixed-epithelioid sarcomatoid). Crystalloids were found in 9 (15\%) of the 59 epithelioid mesotheliomas, indicating that these structures are not as rare as had been thought. That these inclusions were demonstrated in tumors exhibiting diverse histological patterns and were not confined to a single subtype of epithelioid mesothelioma indicates that, because of their unique morphology, when present, they can assist in the diagnosis of these tumors. In addition, oncocytic features were also seen in one of the cases with crystalloid inclusions. Pathologists should be aware of the fact that, even though uncommon, mesotheliomas can present oncocytic morphology and, therefore, these tumors should be included in the differential diagnosis of those neoplasms that display similar morphological features, and which can metastasize to the serosal membranes. To my knowledge, an oncocytic mesothelioma has not previously been reported.

Modern Pathology (2012) 25, 272-281; doi:10.1038/modpathol.2011.159; published online 28 October 2011
\end{abstract}

Keywords: crystalloids; electron microscopy; immunohistochemistry; mesothelioma; oncocytic mesothelioma

Crystals and crystalloids have been reported in both intracellular and extracellular locations in a wide variety of tumors. Their presence constitutes an important marker for the diagnosis of certain tumors, such as alveolar soft part sarcomas and juxtaglomerular cell tumors. ${ }^{1-3}$ As a large number of crystalloids were seen in the vast majority of the neoplastic cells in a biopsy of an epithelioid mesothelioma submitted for ultrastructural evaluation, and as these structures have rarely been reported in these tumors, and their value as an ultrastructural marker in this diagnosis has not yet been fully established, I was prompted to search for crystalloids in 69 consecutive mesothelioma cases.

Correspondence: Dr NG Ordóñez, MD, Department of Pathology, The University of Texas MD Anderson Cancer Center, 1515 Holcombe Boulevard, Houston, TX 77030, USA.

E-mail: nordonez@mdanderson.org

Received 3 August 2011; revised 26 August 2011; accepted 26 August 2011; published online 28 October 2011
The purpose of this article is to provide a detailed ultrastructural description of nine epithelioid mesotheliomas containing abundant crystalloid inclusions, including one that also exhibited oncocytic features. Pathologists need to be aware that, because of their unique morphology, these structures can serve as a useful ultrastructural marker of mesothelioma. Additionally, given that mesotheliomas can present oncocytic morphology, they should be included in the differential diagnosis of tumors that exhibit similar morphology and can metastasize to the serosal membranes. To my knowledge, oncocytic mesotheliomas have not previously been documented.

\section{Materials and methods}

Out of the 69 mesotheliomas that were investigated for crystalloids, 59 were diagnosed as epithelioid, 7 as sarcomatoid, and 3 as mixed epithelioid and 
sarcomatoid. Tissue specimens were fixed in $10 \%$ buffered formalin and processed routinely for light microscopy. Sections were cut and stained with hematoxylin-eosin, Mayer's mucicarmine, and Alcian blue ( $\mathrm{pH}$ 2.5), and periodic acid Schiff (PAS) with and without diastase digestion. Samples for electron microscopy were fixed in $2 \%$ glutaraldehyde in phosphate buffer, post-fixed in $1 \%$ osmium tetroxide, and embedded in Epon epoxy resin. Ultrathin sections were stained with uranyl acetate and lead citrate. An average of four EM blocks was studied for each case. Immunohistochemical studies were performed using the avidin-biotin-peroxidase complex method in a Dako Autostainer (Carpinteria, CA, USA). The primary antibodies used are listed in Table 1 . The immunoperoxidase staining was done using the LSAB2 peroxidase kit (Dako). To enhance the immunostaining, a heat epitope retrieval procedure was performed using a Black-and-Decker vegetable steamer (Shelton, CT, USA). Briefly, deparaffinized sections were placed in a thermoresistant container filled with a buffer solution. Depending upon the antibody, the buffer solutions used were either sodium citrate buffer ( $\mathrm{pH}$ 6.0) or a 1:10 solution of Tris-EDTA buffer ( $\mathrm{pH}$ 8.0). The antigen-antibody reaction was visualized using either $3,3^{\prime}$-diaminobenzidine or 3-amino-9-ethylcarbazole as chromogen. To evaluate the specificity of the antibody, known positive and negative tissues were used as controls.

\section{Results}

\section{Clinical Findings}

Seven patients were male and two were female, ranging in age from 53 to 74 years (mean, 61 years). There was a history of asbestos exposure in six patients and smoking in five patients. In all of the cases, the mesothelioma originated in the pleura. Seven of the patients died of disease 5 to 55 months (mean, 17 months) after diagnosis, one patient is alive with no evidence of disease at 60 months, and there was no follow-up information available for the remaining patient. Clinical information is summarized in Table 2.

\section{Light Microscopic Findings}

Most of the tumors were composed of cuboidal cells, often arranged in a tubulopapillary or solid pattern (Figures 1a-d). The vast majority of the neoplastic cells had eosinophilic cytoplasm in most of the cases; but in three, there were focal areas in which the cells exhibited clear or finely vacuolated cytoplasm (Figure 1a). In one case (case 3), some of the cells had signet ring-like morphology (Figures 1e and $\mathrm{f}$ ), and in another (case 5), the tumor was primarily composed of confluent sheets of polygonal cells with abundant dense eosinophilic cytoplasm exhibiting oncocytoid features (Figures $1 \mathrm{~g}$ and $\mathrm{h}$ ). In two of the cases, there were areas in which the

Table 1 Antibodies used in this study

\begin{tabular}{lllcc}
\hline Marker & Source & Type & Dilution & Antigen retrieval \\
\hline Calretinin & Zymed, South San Francisco, CA, USA & PAb (rabbit) & $1: 20$ & Yes (citrate) \\
CD15 & Becton-Dickinson, Mountainview, CA, USA & Leu-M1 MAb & $1: 40$ & Yes (Tris-EDTA) \\
CEA & NeoMarkers, Fremont, CA, USA & PAb (rabbit) & $1: 175$ & No \\
Keratin 5/6 & Dako Corporation, Carpinteria, CA, USA & D5/16B4 MAb & $1: 100$ & Yes (citrate) \\
MOC-31 & Dako Corporation & MAb & $1: 50$ & Yes (citrate) \\
Podoplanin & Signet Laboratories, Dedham, MA, USA & D2-40 MAb & $1: 25$ & Yes (Tris-EDTA) \\
TAG-72 & BioGenex, San Ramon, CA, USA & B72.3 MAb & $1: 300$ & No \\
WT1 & Dako Corporation & 6F-H2 MAb & $1: 40$ & Yes (Tris-EDTA) \\
\hline
\end{tabular}

Abbreviation: $\mathrm{CEA}=$ carcinoembryonic antigen

Table 2 Summary of clinical findings

\begin{tabular}{llcclll}
\hline Case & $\begin{array}{c}\text { Sex/ } \\
\text { age }\end{array}$ & $\begin{array}{c}\text { Asbestos } \\
\text { exposure }\end{array}$ & $\begin{array}{c}\text { History of } \\
\text { smoking }\end{array}$ & Location & Treatment & Follow-up (months) \\
\hline 1 & $\mathrm{M} / 53$ & No & Yes & Rt pleura & Experimental protocol & DOD (12) \\
2 & $\mathrm{M} / 60$ & Yes & Yes & Rt pleura & Pneumonectomy & DOD (5) \\
3 & $\mathrm{M} / 67$ & Yes & Yes & Rt pleura & Chemotherapy & DOD (18) \\
4 & $\mathrm{~F} / 55$ & No & No & Lt pleura & Pneumonectomy+radiation & DOD (10) \\
5 & $\mathrm{M} / 57$ & Yes & Yes & Rt pleura & Decortication+chemotherapy & DOD (17) \\
6 & $\mathrm{M} / 74$ & No & No & Lt pleura & INA & INA \\
7 & $\mathrm{M} / 60$ & Yes & Yes & Rt pleura & Experimental protocol & DOD (55) \\
8 & $\mathrm{~F} / 54$ & Yes & No & Rt pleura & Chemotherapy+pneumonectomy+radiation & NED (60) \\
9 & $\mathrm{M} / 72$ & Yes & No & Lt pleura & Chemotherapy & DOD (5)
\end{tabular}

Abbreviations: $\mathrm{DOD}=$ died of disease; INA = information not available; NED = no evidence of disease. 


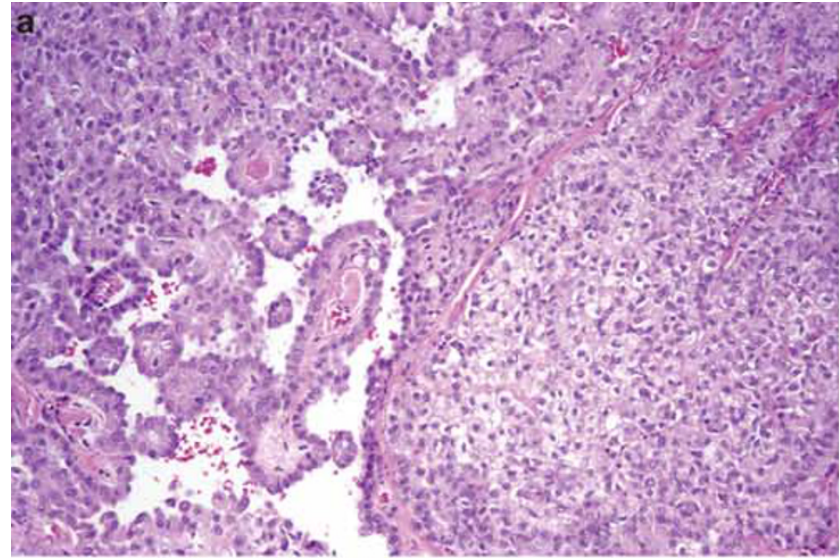

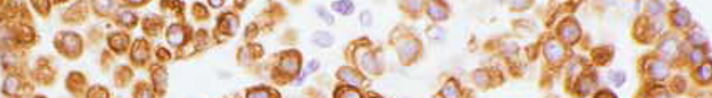

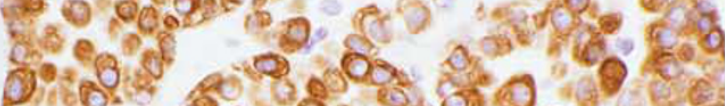

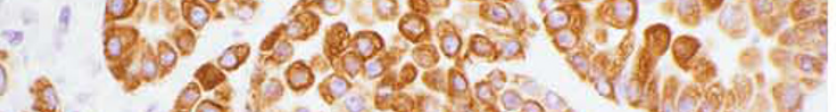

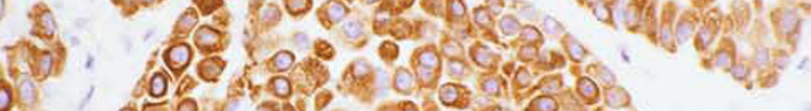

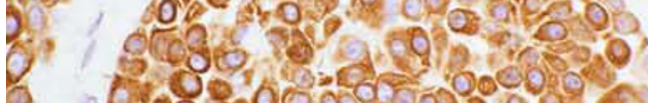

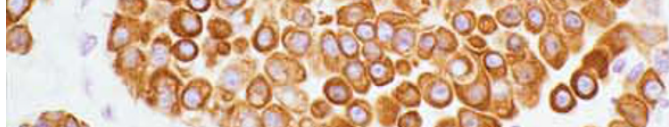

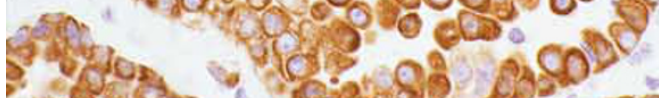

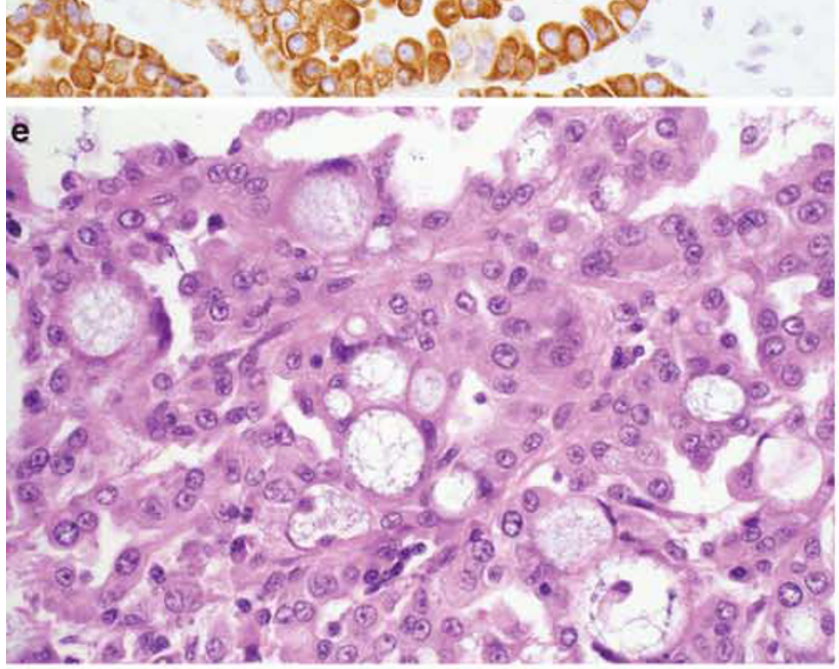

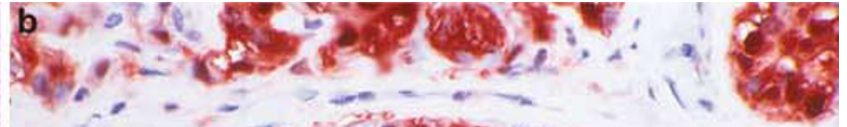

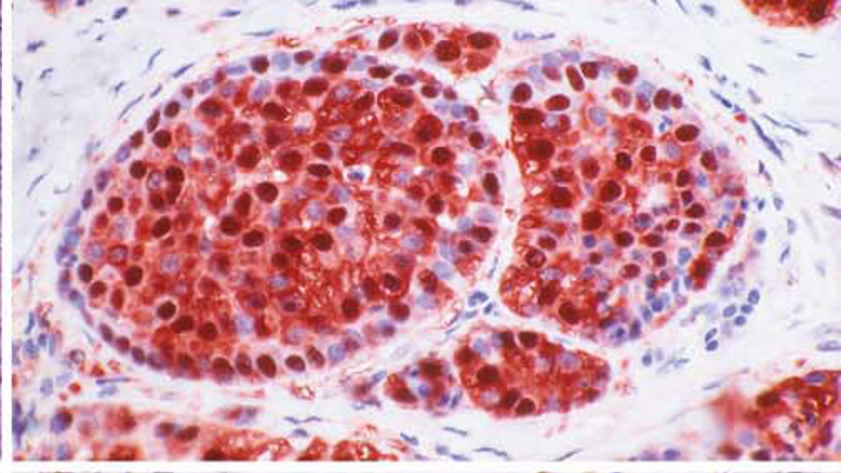

d) तो -

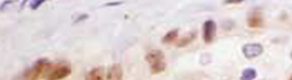

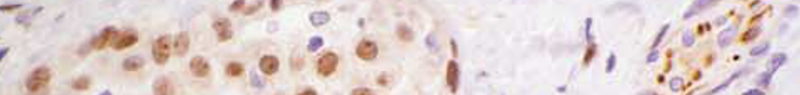

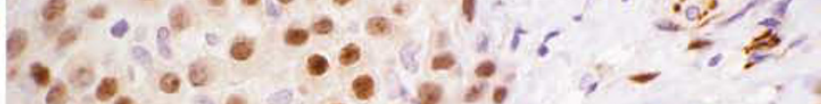

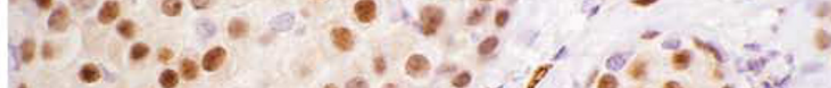

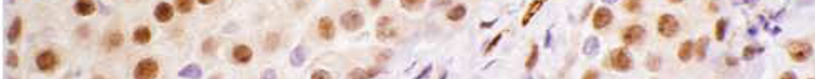

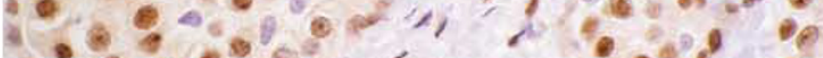

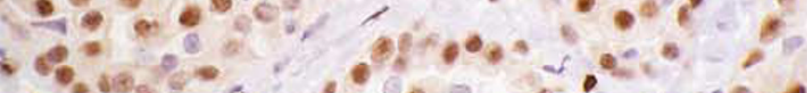
a

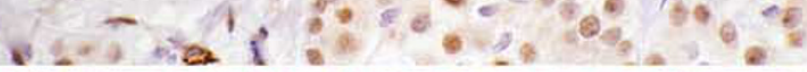

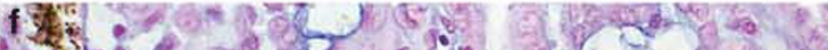

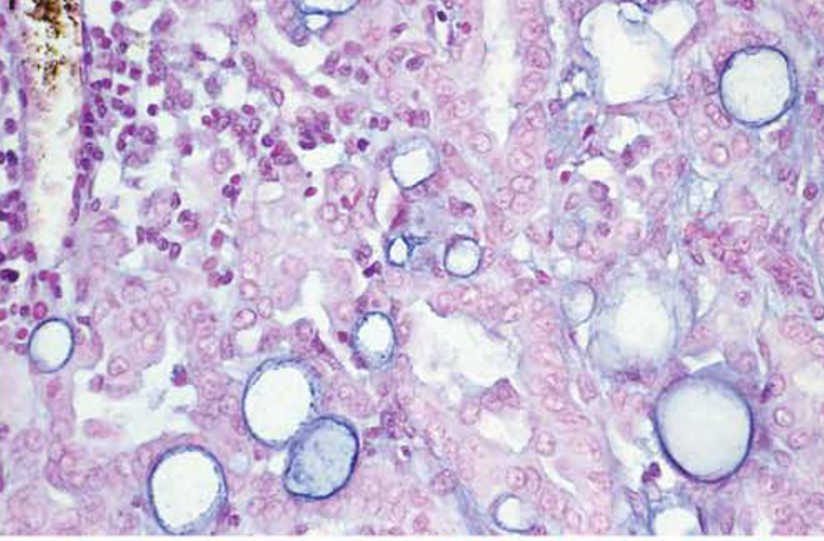

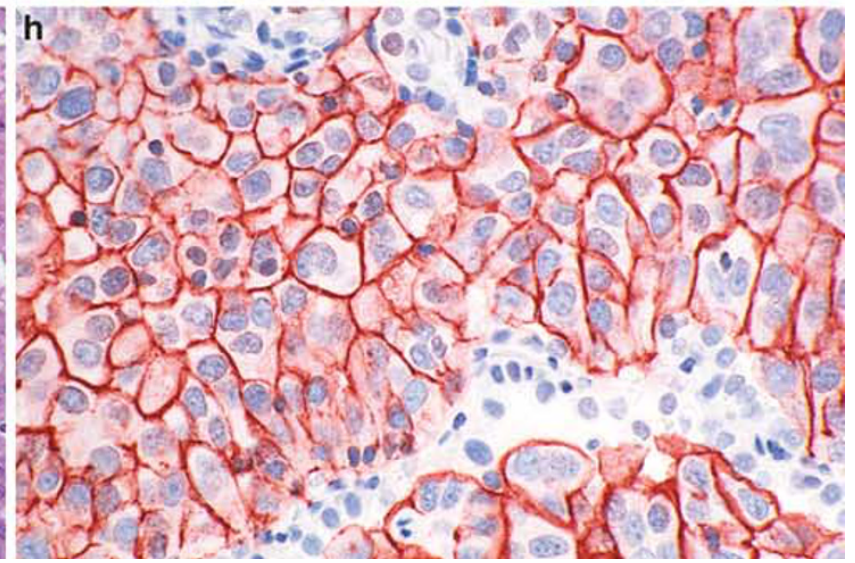


stroma exhibited prominent myxoid changes. PAS stain demonstrated the presence of moderate amounts of glycogen in one case. Small amounts of intracytoplasmic PAS-positive material after diastase digestion were seen in three cases. Alcian blue stain was positive in four cases (Figure 1f). None of the cases were mucicarmine-positive. The most significant light microscopic findings are summarized in Table 3.

\section{Immunohistochemical Findings}

Immunohistochemical studies showed diffuse, strong reactivity for calretinin and podoplanin in the neoplastic cells in all nine cases. The staining for calretinin was both cytoplasmic and nuclear (Figure 1b); whereas, that for podoplanin was membranous and occurred mainly along the apical surface of the cells (Figure 1h). In addition, cytoplasmic staining for keratin 5/6 and nuclear positivity for WT-1 was observed in seven of seven cases and in six of eight cases stained for these markers, respectively (Figures 1c and d).
No reactivity was seen for CEA, TAG-72 (B72.3), MOC-31, or CD15.

\section{Electron Microscopic Findings}

Ultrastructural studies demonstrated that the neoplastic cells rested on a basal lamina and that the neighboring cells were often joined by well-developed desmosomes. The apical surfaces were often covered by a large number of long, slender, branching microvilli. The most common striking feature in all of the cases, however, was the presence of large numbers of crystalloids that, in some cells, were found primarily or exclusively within the cell cytoplasm, whereas in others, they were extracellular (Figures 2a and b). The most common type of intracytoplasmic crystalloids consisted of rigid, hollow tubules with a honeycomb structure that were located within the cisternae of rough endoplasmic reticulum (Figures $3 \mathrm{a}, \mathrm{b}$ and 4). Although these were observed in six cases, they were particularly abundant in case 1, in which they were identified in the majority of the neoplastic cells

Table 3 Summary of pathology findings

\begin{tabular}{llll}
\hline Case & Type of specimen & Light microscopy & Electron microscopy \\
\hline 1 & Pleural biopsy & Papillary and solid with focal areas of clear cells & Numerous intracytoplasmic tubular crystalloids. \\
2 & Pneumonectomy & $\begin{array}{l}\text { Cuboidal cells arranged in a mixed papillary and } \\
\text { solid pattern. Most cells had eosinophilic } \\
\text { cytoplasm, but in some areas, it was clear or } \\
\text { vacuolated. }\end{array}$ & $\begin{array}{l}\text { Multilayered crystalloid material with rough } \\
\text { endoplasmic reticulum. Large number of } \\
\text { extracellular tubular crystalloids. Abundant } \\
\text { intracytoplasmic glycogen. }\end{array}$
\end{tabular}

$3 \quad$ Pleural biopsy

4

Pneumonectomy

Pleural biopsy

$6 \quad$ Pleural biopsy

$7 \quad$ Pleural biopsy

8

Pneumonectomy

9

Pleural biopsy
Large cells with abundant cytoplasm and myxoid stroma. Some cells had signet-ring-like features.

Cuboidal cells arranged in solid sheets or forming papillary structures. The cytoplasm was finely vacuolated or clear. On occasion, some cells contained large intracytoplasmic vacuoles.

Confluent sheets of polygonal cells with dense eosinophilic cytoplasm exhibiting oncocytoid features.

Solid sheets of polygonal cells with clear cytoplasm.

Cuboidal cells arranged in a tubulopapillary or solid pattern.

Cuboidal eosinophilic cells arranged in a solid or tubulopapillary pattern. The stroma was myxoid in some areas.

Polygonal eosinophilic cells arranged in a solid pattern.
Abundant electron-dense material along the apical cell membrane, sometimes containing tubular crystalloids.

Numerous extracellular tubular crystalloids in association with the microvilli in dilated intercellular spaces, as well as with intracytoplasmic lumens.

Intracytoplasmic tubular crystalloids. Numerous closely-packed mitochondria.

Extracellular membranous crystalloid material.

Extracellular tubular crystalloids.

Large amounts of extracellular and intracellular, multilayered crystalloid material. Abundant intracytoplasmic glycogen.

Large amounts of intracellular and extracellular multilayered crystalloid material.

Figure 1 Case 1. (a) The tumor cells are arranged in a papillary and solid pattern, and most have clear cytoplasm. (b) Immunohistochemical preparation showing strong nuclear and cytoplasmic positivity for calretinin. (c) Positive staining for keratin 5/6. (d) Nuclear reactivity for WT1. Case 3. (e) Area of the tumor in which the neoplastic cells show signet-ring-like features. (f) A thin rim of Alcian blue positivity is seen along the lumens of the signet-ring cells. Case 5. (g) Mesothelioma with oncocytic features in which the neoplastic cells show dense eosinophilic cytoplasm. (h) Podoplanin reactivity along the cell membrane. 

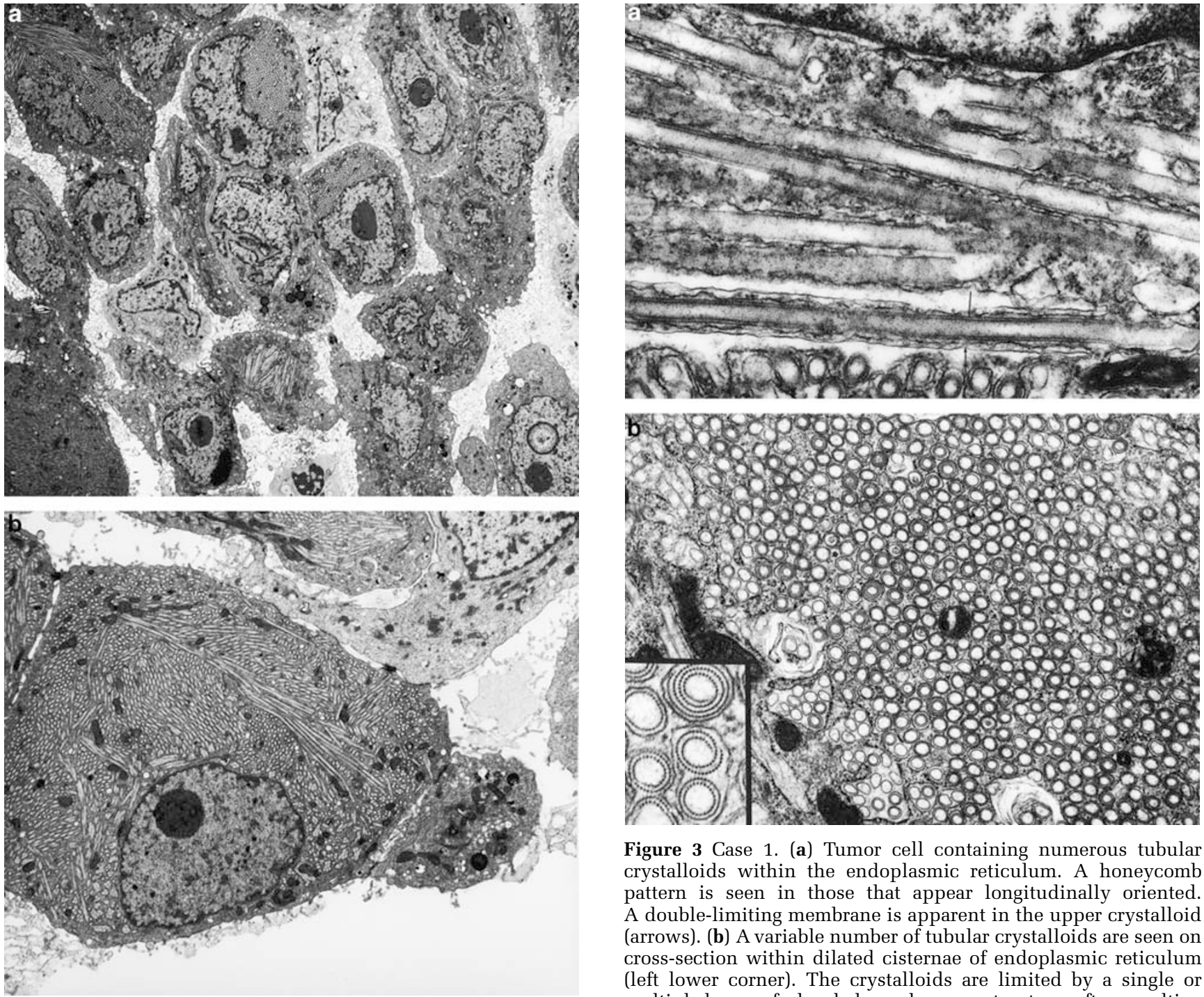

Figure 3 Case 1 . (a) Tumor cell containing numerous tubular crystalloids within the endoplasmic reticulum. A honeycomb pattern is seen in those that appear longitudinally oriented. A double-limiting membrane is apparent in the upper crystalloid (arrows). (b) A variable number of tubular crystalloids are seen on cross-section within dilated cisternae of endoplasmic reticulum (left lower corner). The crystalloids are limited by a single or multiple layers of a beaded membranous structure, often resulting

Figure 2 Case 1. (a) Most of the cells seen in this electron micrograph, which shows the general appearance of the tumor, contain numerous intracytoplasmic crystalloid inclusions. (b) Higher magnification of one of the tumor cells showing the cytoplasm packed with crystalloids $(\mathbf{a}, \times 4000 ; \mathbf{b}, \times 8000)$.

(Figures 2a and b). Although much more limited in number, the same type of crystalloid material was also present in the mesothelioma that exhibited oncocytoid features (case 3, Figures 5a and b).

In two of the cases, in addition to the tubular crystalloids, a multilayered crystalloid material was also observed within the rough endoplasmic reticulum that appeared as a curved, scroll-like, or circular array frequently encircling the tubular crystalloids (Figures 6a and b). In another case, however, the multilayered crystalloids appeared as straight, rigid structures, occupying almost the entire cytoplasmic area in some cells (Figure 7a). Tubular crystalloids were not found in this case.

Crystalloids were also occasionally seen in extracellular locations. In some cases, they appeared as tubular structures, whereas in others, they had in a target-like or spiral configuration (inset; $\mathbf{a}, \times 58700$; $\mathbf{b}$, $\times 38000$; inset, $\times 120000)$

a multilayered configuration (Figures $7 \mathrm{~b}$ and 8). Extracellular tubular crystalloids were found in four cases. One of these cases was characterized by the presence of large amounts of medium electrondense material, presumably hyaluronic acid, or hyaluronic acid containing proteoglycans in which the tubular crystalloids appeared to be embedded (Figure 9). Multilayered crystalloids were found in the interstitium where they appeared as straight, rigid structures (Figure $7 \mathrm{~b}$ ), or in close relation to microvilli, sometimes giving the impression of coating these structures (Figures 10a and b).

\section{Discussion}

Crystals and crystalloids are relatively uncommon in tumors and when they occur, they are usually of interest to pathologists, not only because of their striking morphology, but also because they may 


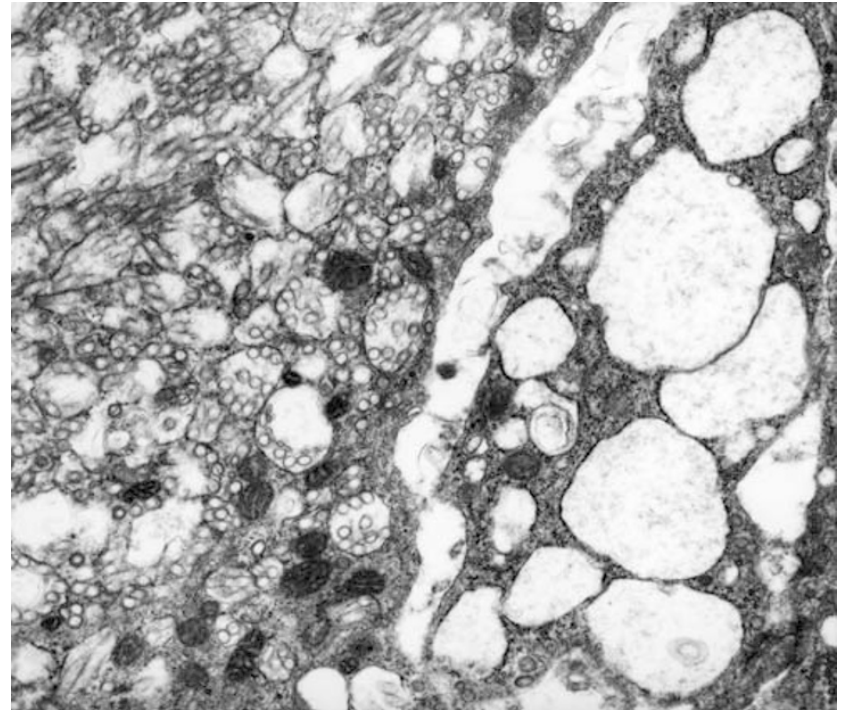

Figure 4 Case 1. Portion of two cells showing marked dilatation of the endoplasmic reticulum that, in the cell on the left, appears to be filled with crystalloids $(\times 24000)$.

serve as tumor markers in some instances. The type of crystals described in the present investigation has only rarely been reported, and neither their relationship to the various histological types of mesotheliomas nor their frequency have been investigated. In 1996, Hammar et $a l^{4}$ reported four cases of mucicarmine-positive epithelioid mesothelioma with crystallized material associated with microvilli that, on cross section, had a hollow tubular appearance. This crystallized material was also noted in the cytoplasm of one of the cases. The authors also described one case with another type of crystalloid inclusion consisting of complex multilayered sheaves within the cisternae of the endoplasmic reticulum. These inclusions, which had previously been described by Henderson et $a l^{5}$ in the neoplastic cells of a pleural effusion from a patient with mesothelioma, were thought to be secretory products of the rough endoplasmic reticulum that could not be transported and secreted by the cell. Since the publication of the two previously mentioned studies, only one additional case of an epithelioid mesothelioma with large amounts of multilayered intracytoplasmic crystalloid inclusions has been reported. ${ }^{6}$ According to the authors of this report, crystalloid inclusions in mesotheliomas are very rare, as they found them in only 1 of the 200 mesotheliomas they had studied by electron microscopy. That I was able to find crystalloid inclusions in 9 of 69 consecutive mesothelioma cases indicates that these structures are not as rare as was previously thought.

Histologically, all of the cases in which crystalloids were found were epithelioid mesotheliomas. As they were found in all histological subtypes of these tumors, their presence indicates that they could serve as a broad marker of mesothelioma.
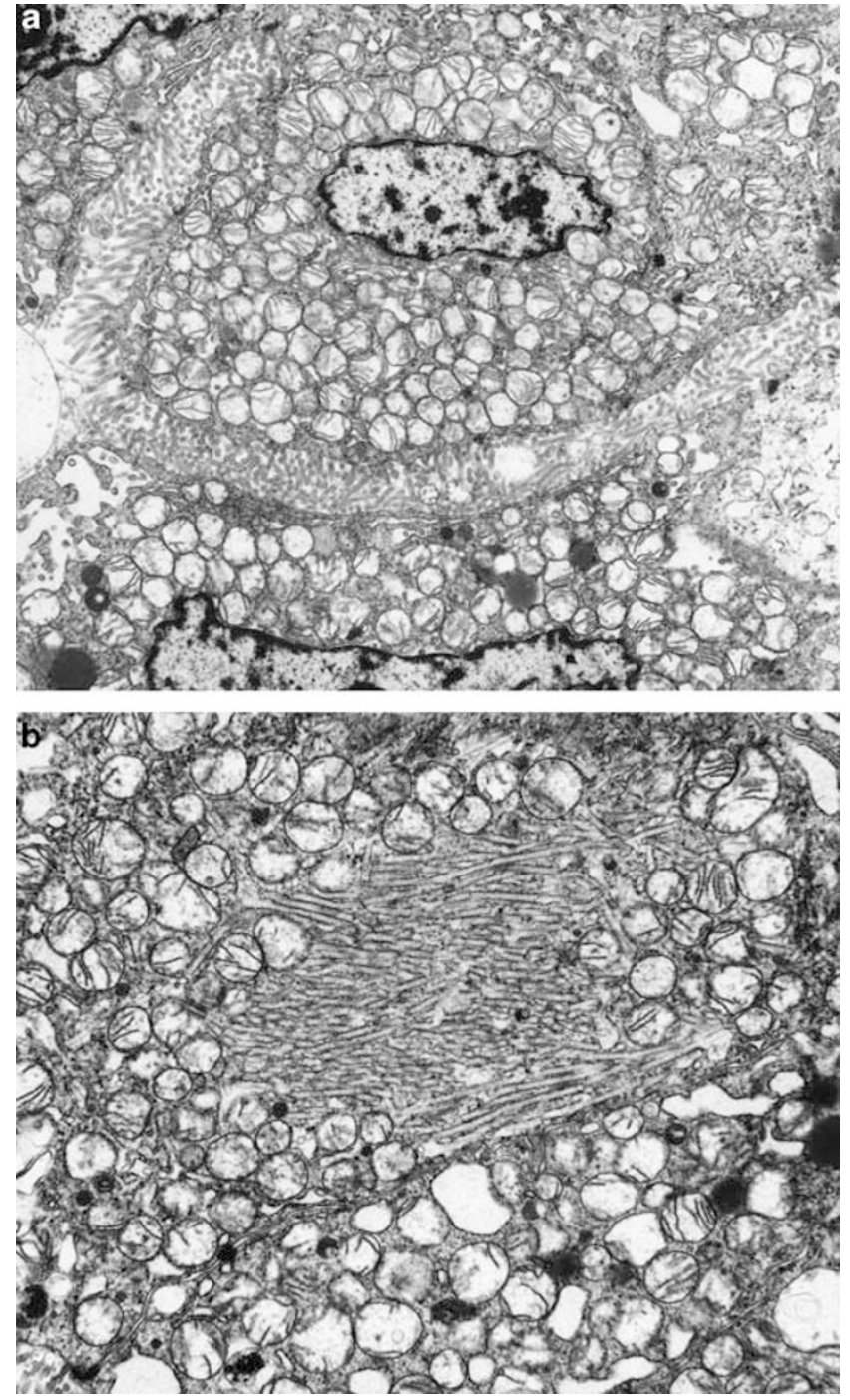

Figure 5 Case 5. (a) Mesothelioma with oncocytic features demonstrating that the cytoplasm of the tumor cells is filled with mitochondria and the cell membrane is covered by numerous long microvilli. (b) Portion of a cell packed with mitochondria showing numerous tubular crystalloids $(\mathbf{a}, \times 8000 ; \mathbf{b}, \times 15000)$.

Even though no crystalloids were found in any of the seven sarcomatoid mesotheliomas investigated, this finding does not necessarily indicate that these structures do not occur in this type of tumor as the number of cases examined was too small to draw this conclusion.

In contrast to Hammar et $a l^{4}{ }^{4}$ who reported mucicarmine positivity in all four of their mesothelioma cases with crystalloids similar to those seen in the present series, none of the cases in the current study were mucicarmine positive. The cause of the differences in these results is unclear. Because Alcian blue positivity was observed along the lumens of the signet-ring cells in the cases that also showed medium electron-dense material in that location (case 3, Figures 1f and 9), and this staining was markedly reduced after pretreatment with hyaluronidase, demonstrates that the material most 

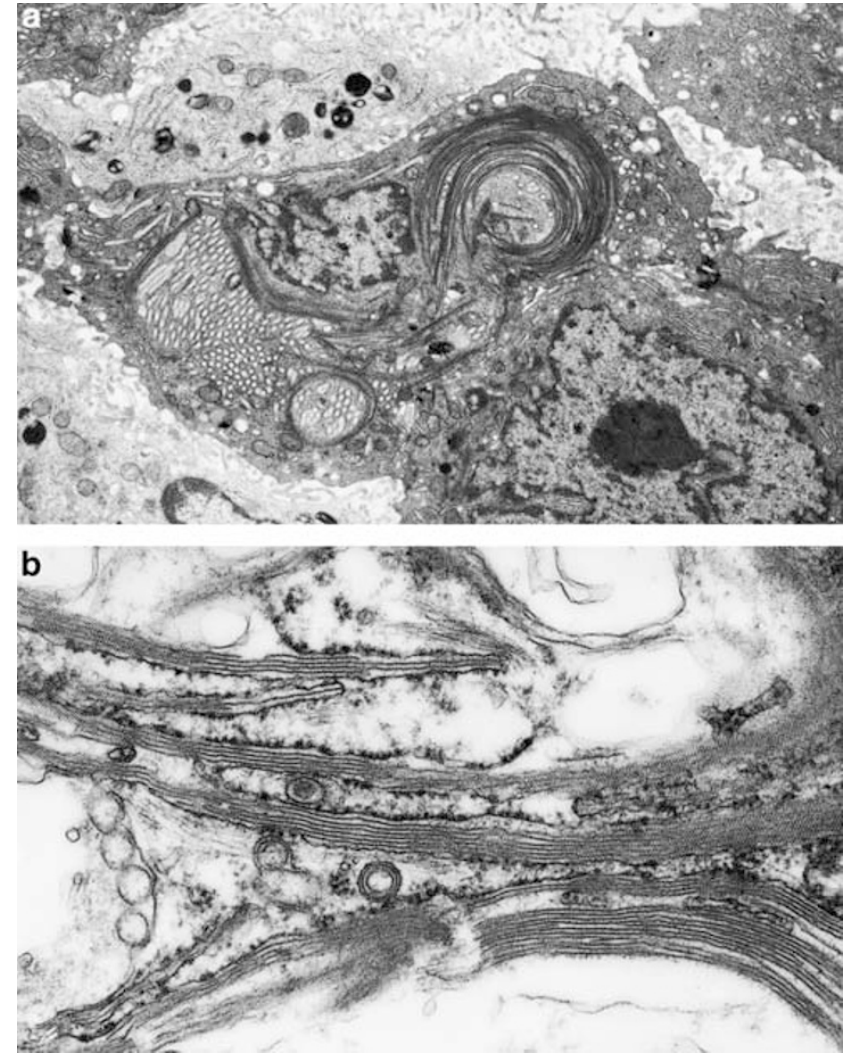

Figure 6 Case 2. (a) A multilayered inclusion arranged in a scrolllike array surrounding tubular crystalloids. (b) Higher magnification demonstrating the intracisternal location of the multilayered crystalloids. A fine cross-periodicity is evident on the right $(\mathbf{a}, \times 10000 ; \mathbf{b}, \times 63000)$.

likely represents proteoglycan. ${ }^{4}$ That the case in which intracytoplasmic crystalloids were seen in most of the neoplastic cells (case 1) was Alcian bluenegative indicates that the tubular crystalloids may not represent crystallized proteoglycan, as has been previously suggested. ${ }^{4}$ This case, however, did demonstrate a minute amount of intracytoplasmic, PAS-positive material after diastase digestion. As the crystalloids seen by electron microscopy were quite numerous, whereas the PAS-positive material was extremely scanty, it is unclear as to whether this material actually corresponds to the crystalloids.

Electron microscopy was once regarded as the 'gold standard' in the diagnosis of mesothelioma. This technique has seen a general decline in usage in recent years, because of the supposed expenses involved and the specialized knowledge required to interpret the results, and it has largely been replaced by immunohistochemistry. Even though a large number of immunohistochemical markers that can be used to assist in distinguishing between epithelioid mesotheliomas and carcinomas are currently available, none of them is absolutely specific and, therefore, the diagnosis of mesothelioma is based on the use of panels composed of markers that are frequently expressed in mesotheliomas, but not in carcinomas (positive mesothelioma markers) and
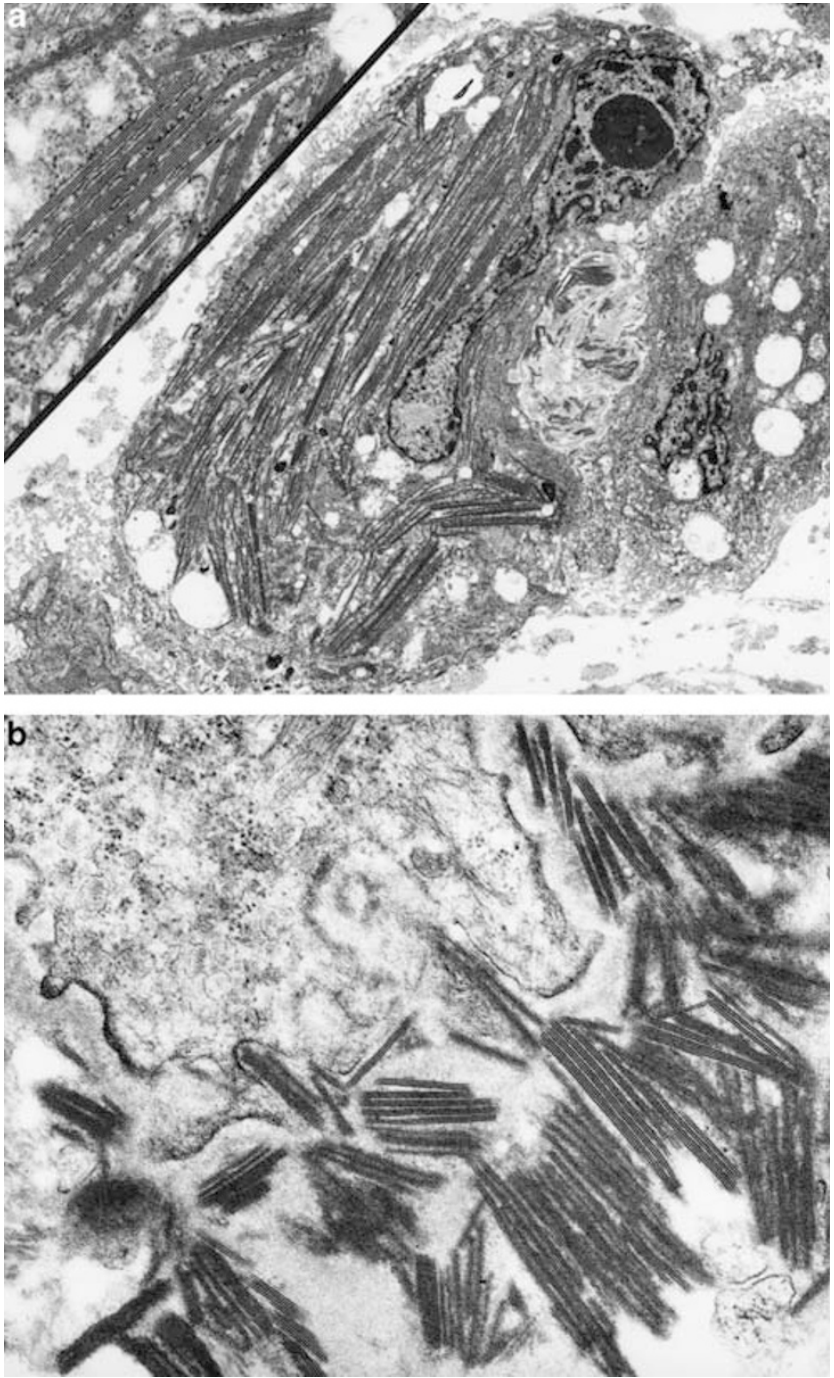

Figure 7 Case 9. (a) Tumor cell filled with large amounts of straight, multilayered crystalloid material, which, at higher magnification (inset), is located within the rough endoplasmic reticulum. (b) Multilayered crystalloids are present in the interstitium $(\mathbf{a}, \times 7200$, inset, $\times 24000 ; \mathbf{b}, \times 48000)$.

those that are commonly expressed in carcinomas, but not in mesotheliomas (negative mesothelioma markers)..$^{7,8}$ In some instances, however, due to the aberrant expression of some of these markers or the presence of other unexpected findings, such as the presence of mucicarmine positivity in a mesothelioma, the use of electron microscopy becomes critical to establish the correct diagnosis. Traditionally, mucicarmine positivity has been regarded as a characteristic finding in adenocarcinoma that, when present, can help in distinguishing these tumors from mesotheliomas, which are generally considered to be mucicarmine-negative. Hammar et $a l,{ }^{4}$ however, demonstrated that mesotheliomas can be mucicarmine-positive, even though this is an uncommon finding. In addition to being mucicarmine-positive, all four of their mesothelioma cases with crystalloids were also 


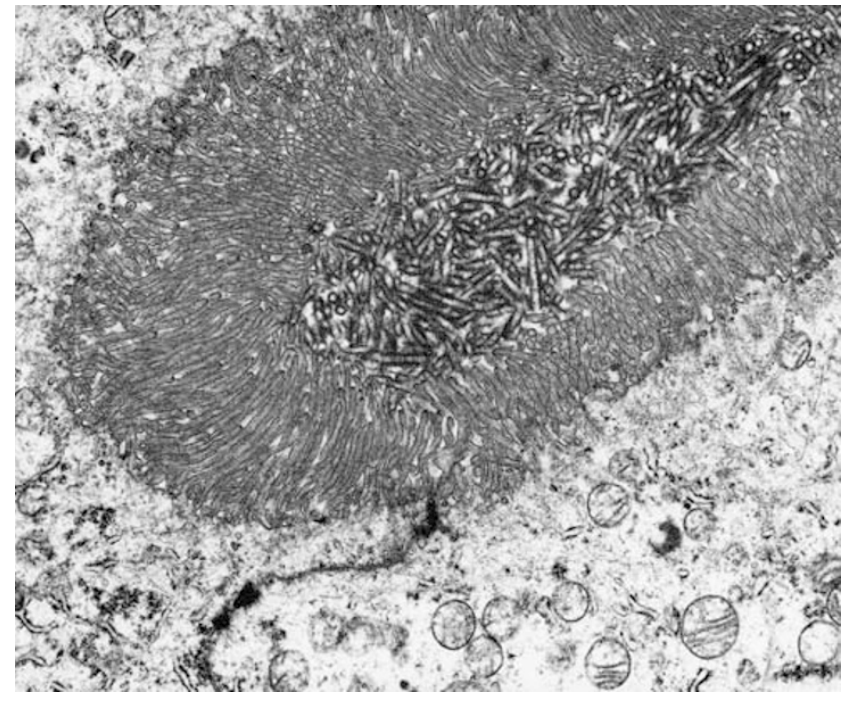

Figure 8 Case 7. Numerous tubular crystalloids appear to obliterate a tubular structure, whose apical surface is covered by abundant slender microvilli $(\times 16000)$.

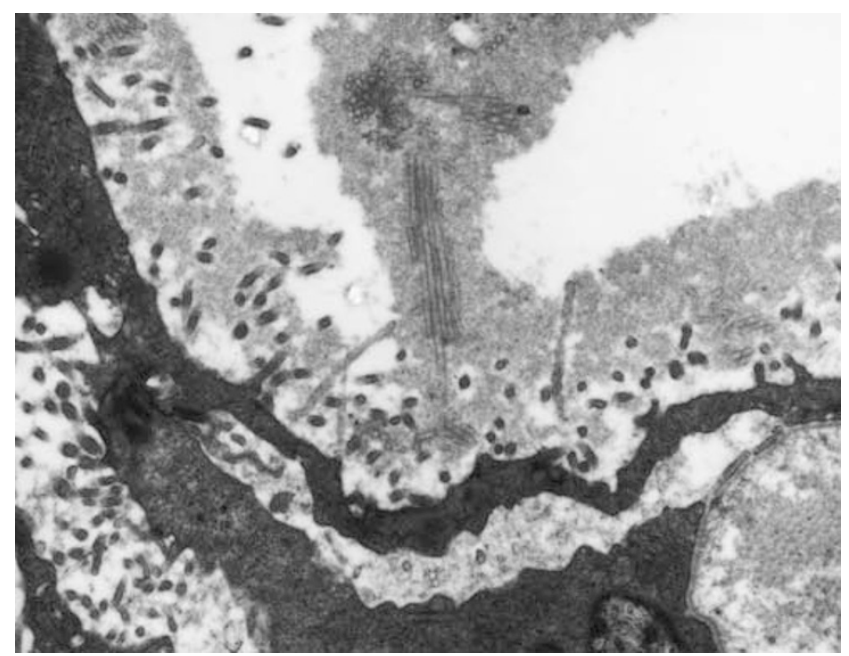

Figure 9 Case 3. Portion of a lumen showing large amounts of medium electron-dense material and tubular crystalloids $(\times 18400)$.

CEA-positive. As the intensity of the reaction was reduced or eradicated by pretreatment with hyaluronidase, the authors suggested that hyaluronic acid may have been responsible for the CEA positivity. Additionally, they reported focal CD15, B72.3, and Ber-EP4 positivity in one of the cases in which the aberrant reaction was also affected by treatment with hyaluronidase. In contrast to Hammar et $a l^{4}$ cases, none of the mesotheliomas in the present series exhibited CEA expression, a finding that suggests that the CEA positivity seen in Hammar et al ${ }^{4}$ cases most likely represents a cross-reactivity of the polyclonal antibody used in that study. Although none of the immunohistochemical markers investigated in the present series were found to be aberrantly expressed in any of the cases, aberrant expression of most of these markers, including
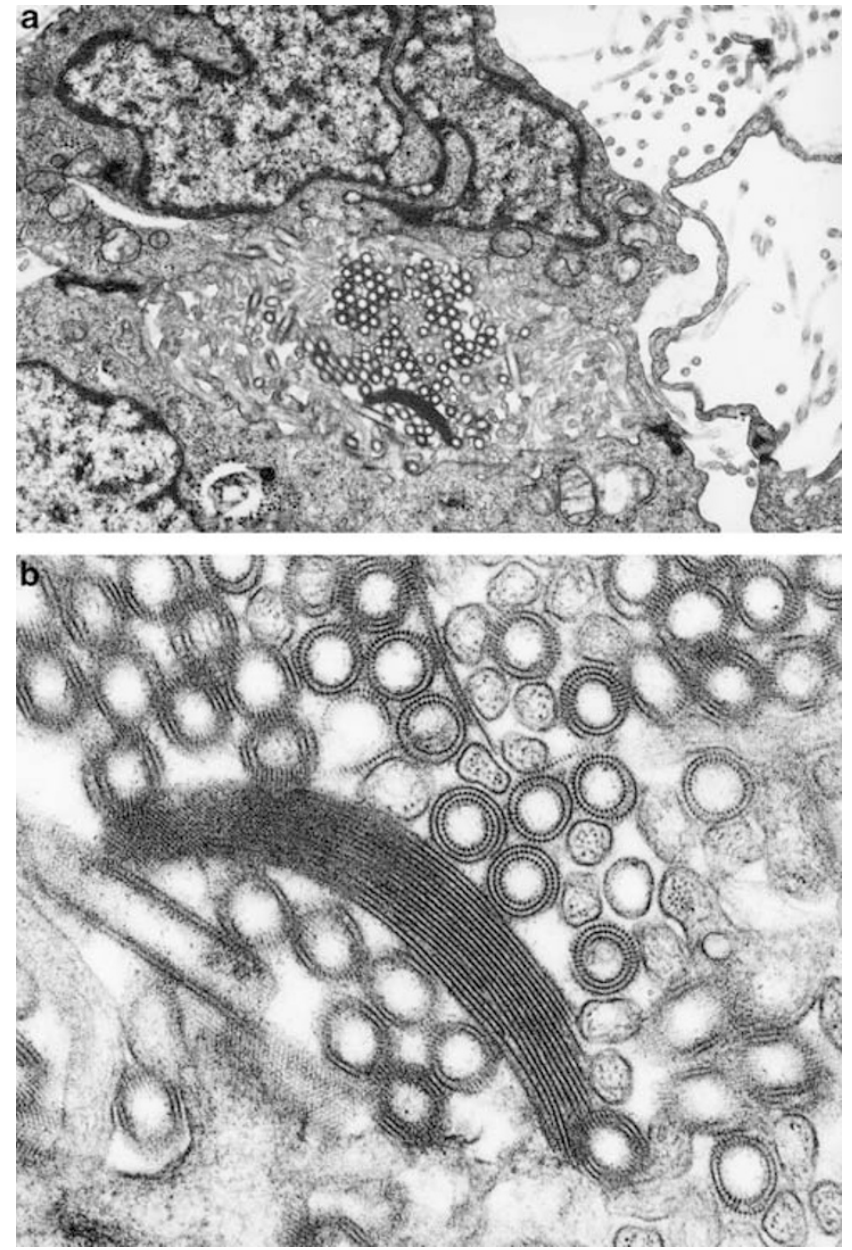

Figure 10 Case 2. (a) Dilated intercellular space containing multilayered crystalloid material. (b) Higher magnification showing crystalloid material that appears to possibly be coating some microvilli (right lower corner). A punctuate substructure of the crystalloid material is apparent $(\mathbf{a}, \times 16800 ; \mathbf{b}, \times 108000)$.

CD15, B72.3, and Ber-EP4, has been documented in mesotheliomas in the literature, ${ }^{9}$ thus demonstrating the need for both electron microscopy and the recognition of new ultrastructural markers for mesothelioma.

One of the cases in the present study that merits further discussion is the mesothelioma with oncocytic features. Malignant oncocytic tumors are relatively uncommon and, to my knowledge, they have not been documented as arising in the serosal membranes, although they have been reported in a variety of other organs, including the thyroid, ${ }^{10}$ pancreas, ${ }^{11}$ adrenal gland, ${ }^{12}$ and prostate. ${ }^{13}$ Mesotheliomas primarily composed of large polygonal cells having deeply eosinophilic cytoplasm are rare and have been reported under the designation deciduoid mesothelioma. ${ }^{14}$ In these cases, the cytoplasmic changes are the result of the accumulation of large amounts of intermediate filaments. Histologically, the pleural tumor in the present study was made of large polygonal cells with dense eosinophilic cytoplasm that, by electron microscopy, was 
demonstrated to be the result of the accumulation of a large number of mitochondria.

The differential diagnosis of epithelioid mesothelioma with oncocytic features includes a wide variety of carcinomas made of large polygonal cells with deeply eosinophilic cytoplasm. ${ }^{15}$ One of the light microscopic findings that can assist in the diagnosis of oncocytic tumors is the cytoplasmic granularity, but this feature is not always related to the presence of mitochondria, as it can occur as a result of the cytoplasmic accumulation of a large number of neurosecretory granules, as has been reported to occur in some medullary carcinomas of the thyroid, ${ }^{15}$ or lysosomes, as seen in granular cell tumors. ${ }^{16}$ Several anti-mitochondrial antibodies, such as the mES-13 and the 113-1 mouse monoclonal antibodies, are commercially available and can be successfully used on routinely fixed and processed specimens. These antibodies have been used in the demonstration of mitochondria in normal and neoplastic tissues, including mitochondria-rich tumors. ${ }^{17,18}$ Although the oncocytic tumors in these studies have been reported to be invariably, and often strongly, positive, some cases have been reported in which there were a significant number of cells that were only weakly positive or even negative. ${ }^{17}$ Furthermore, it should be pointed out that, although some tumors may exhibit strong reactivity with an anti-mitochondrial antibody, and have a relatively large number of mitochondria, the number of mitochondria might not be sufficient to fulfill the criteria for a diagnosis of an oncocytic tumor. Because of this, electron microscopy remains the best and most accurate technique for confirming the oncocytic nature of a tumor by providing a direct observation of the number of mitochondria present in the neoplastic cells. In the present case, cytoplasmic granularity was not apparent on the hematoxylin-eosin-stained sections; therefore, electron microscopy proved to be critical in determining the oncocytic character of the tumor. It should be mentioned that, in some instances, the ultrastructural features of these organelles may provide further insight into the diagnosis of the tumor. For example, the demonstration of mitochondria with tubular cristae in the eosinophilic-rich variant of chromophobe carcinoma of the kidney is a valuable ultrastructural finding that can assist in distinguishing these tumors from both renal oncocytomas and the granular cell variant of conventional renal cell carcinoma. ${ }^{19}$

In conclusion, the crystalloid inclusions described in this study are not a rare finding in mesotheliomas, and because of their unique morphology, when present, they can serve as an ultrastructural marker of mesothelioma. The case with oncocytoid features illustrates that not only is electron microscopy still helpful in the diagnosis of mesotheliomas, but it also provides a better understanding of the morphological features observed by light microscopy.

\section{Disclosure/conflict of interest}

The author declares no conflict of interest.

\section{References}

1 Camilleri JP, Hinglais N, Bruneval P, et al. Renin storage and cell differentiation in juxtaglomerular cell tumors: an immunohistochemical and ultrastructural study of three cases. Hum Pathol 1984;15:1069-1079.

2 Hasegawa A. Juxtaglomerular cells tumor of the kidney: a case report with electron microscopic and flow cytometric investigation. Ultrastruct Pathol 1997; 21:201-208.

3 Ordóñez NG, Mackay B. Alveolar soft-part sarcoma: a review of the pathology and histogenesis. Ultrastruct Pathol 1998;22:275-292.

4 Hammar SP, Bockus DE, Remington FL, et al. Mucinpositive epithelial mesotheliomas: a histochemical, immunohistochemical, and ultrastructural comparison with mucin-producing pulmonary adenocarcinomas. Ultrastruct Pathol 1996;20:293-325.

5 Henderson DW, Shilkin KB, Whitaker D, et al. The pathology of malignant mesothelioma, including immunohistology and ultrastructure. In: Henderson DW, Shilkin KG, Langlois SLD, Whitaker D (eds). Malignant Mesothelioma. Hemisphere: New York, 1992, pp 69-139.

6 Goedhals J, Beukes CA, Cooper S. Malignant mesothelioma with intracytoplasmic crystalline inclusions. Ultrastruct Pathol 2007;31:73-76.

7 Ordóñez NG. What are the current best immunohistochemical markers for the diagnosis of epithelioid mesothelioma? A review and update. Hum Pathol 2007;38:1-16.

8 Husain AN, Colby TV, Ordóñez NG, et al. Guidelines for pathologic diagnosis of malignant mesothelioma: a consensus statement from the International Mesothelioma Interest Group. Arch Pathol Lab Med 2009;133: 1317-1331.

9 Ordóñez NG. The immunohistochemical diagnosis of mesothelioma: a comparative study of epithelioid mesothelioma and lung adenocarcinoma. Am J Surg Pathol 2003;27:1031-1051.

10 Baloch ZW, LiVolsi VA. Oncocytic lesions of the neuroendocrine system. Semin Diagn Pathol 1999;16: 190-199.

11 Adsay NV, Adair CF, Heffess CS, et al. Intraductal oncocytic papillary neoplasm of the pancreas. Am J Surg Pathol 1996;20:980-994.

12 Krishnamurthy S, Ordóñez NG, Shelton TO, et al. Fine-needle aspiration cytology of a case of oncocytic adrenocortical carcinoma. Diagn Cytopathol 2000;22: 299-303.

13 Ordóñez NG, Ro JY, Ayala AG. Metastatic prostatic carcinoma presenting as an oncocytic tumor. Am J Surg Pathol 1992;16:1007-1012.

14 Ordóñez NG. Epithelial mesothelioma with deciduoid features: report of four cases. Am J Surg Pathol 2000; 24:816-823.

15 Nappi O, Ferrara G, Wick MR. Neoplasms composed of eosinophilic polygonal cells: an overview with consideration of different cytomorphologic patterns. Semin Diagn Pathol 1999;16:82-90.

16 Ordóñez NG. Granular cell tumor: a review and update. Adv Anat Pathol 1999;6:186-203. 
17 Weiss LM, Gaffey MJ, Warhol MJ, et al. Immunocytochemical characterization of a monoclonal antibody directed against mitochondria reactive in paraffinembedded sections. Modern Pathol 1991;4:596-601.

18 Tickoo SK, Amin MB, Linden MD, et al. Antimitochondrial antibody (113-1) in the differential diagnosis of granular renal cell tumors. Am J Surg Pathol 1997;21:922-930.

19 Erlandson RA, Shek TWH, Reuter VE. Diagnostic significance of mitochondria in four types of renal epithelial neoplasms: an ultrastructural study of 60 tumors. Ultrastruct Pathol 1997;21:409-417. 